Pacific Journal of Mathematic 


\title{
THE PSEUDO-RADICAL OF A COMMUTATIVE RING
}

\author{
Robert W. Gilmer, JR.
}

If $D$ is an integral domain with identity having quotient field $K$, the pseudo-radical of $D$ is defined to be the intersection of all nonzero prime ideals of $D$. Consideration of the pseudo-radical arises naturally in examining the relation between the statements " $D$ has Jacobson radical zero" and " $D[u]$ has Jacobson radical zero, where $u \in K$ ". Theorem 4 proves that the first statement implies the second. As a corollary it follows that if $M$ is a prime ideal of the polynomial ring $R[X]$ over a commutative ring $R$ and if $P=M \cap R$, then $M$ is an intersection of maximal ideals of $R[X]$ if $P$ is an intersection of maximal ideals of $R$. Consequently, if $R$ is a Hilbert ring, $R[X]$ is also a Hilbert ring. The remainder of the paper is devoted to a study of domains having nonzero pseudo-radical.

Goldman has defined in [6] the concept of a Hilbert ring: the commutative ring $R$ with identity is a Hilbert ring if each proper prime ideal of $R$ is an intersection of maximal ideals; here proper means an ideal different from $R$. The terminology is motivated by the observation that Hilbert's Nullstellensatz may be interpreted as asserting that each proper prime ideal of the polynomial domain $K\left[X_{1}, \cdots, X_{n}\right]$ for $K$ a field, is an intersection of maximal ideals. In work done independently but at approximately the same time, Krull introduced in [10] the concept of a Jacobson ring; the reason for the terminology being obvious from the definition: the commutative ring $R$ with identity is a Jacobson ring if for each proper ideal $A$ of $R$, the radical of $R / A$ and the Jacobson radical of $R / A$ coincide. From these two definitions it is easily seen that $R$ is a Hilbert ring if and only if $R$ is a Jacobson ring. [10; p. 359]. In the remainder of this paper we shall use the term Hilbert ring for the notions described above.

In Theorem 3 of [6], Goldman shows $R$ and the polynomial ring $R[X]$ are simultaneously Hilbert rings. Also, (Theorem 5 of [6]), $R[X]$ is a Hilbert ring if and only if for each maximal ideal $M$ of $R[X], M \cap R$ is maximal in $R$. We originally set out to consider the following "local" case of Goldman's Theorem 3:

Suppose $M$ is a proper prime ideal of $R[X]$ and $P=M \cap R$. What is the relation between the following statements?

(1) $M$ is an intersection of maximal ideals of $R[X]$.

(2) $P$ is an intersection of maximal ideals of $R$.

Corollary 2 shows that (2) implies (1). Goldman's Theorem 3 
follows as a corollary. It can be seen that the above question may be reduced to considering the following problem.

Suppose $D$ is an integral domain with quotient field $K$ and $t \in K$. What is the relation between the statements

(a) the Jacobson radical of $D$ is zero.

(b) the Jacobson radical of $D[t]$ is zero.

Hence, as one might expect from the equivalence of the concepts Hilbert ring and Jacobson ring, we are led into a consideration of the Jacobson radical of a ring and its overrings. For integral domains it becomes natural in this connection to consider the pseudo-radical, defined as follows: If $R$ is a commutative ring with identity, the pseudo-radical of $R$ is the intersection of all nonzero prime ideals of $R$.

Section 2 of this paper lists some known results concerning relations between the ideal theory of $R$ and that of the polynomial ring $R[X]$. Section 3 considers domains $D$ with nonzero pseudo-radical and the effect of this hypothesis on the ideal theory of $D[X]$. In $\S 4$ we consider the italicized questions previously listed.

Our notation and terminology will in general be that of ZariskiSamuel [19] [20]. In particular "ring" always means a commutative ring with identity and it is assumed that each subring contains the identity of the larger ring. We use the following terms which are not found in Zariski-Samuel. If $D$ is an integral domain with quotient field $K$ we say $D$ has the $Q R$-property if each domain between $D$ and $K$ is a quotient ring of $D$ with respect to some multiplicative system in $D$. [5]. If for each prime ideal $P$ of $D$ the quotient ring $D_{P}$ is a valuation ring, we say $D$ is a Prïfer domain. For elementary properties of Prüfer domains see [7]. If each $D_{P}$ is in fact a rank one discrete valuation ring, we say $D$ is almost Dedekind [3].

2. The ideal theory of $R$ and of $R[X]$. The relationship between the ideal theory of a ring $R$ and its polynomial ring $R[X]$ has been studied extensively; for the case when $R$ is Noetherian principally by Krull [10], for $R$ Prüfer by Seidenberg [15], for $R$ Dedekind by Nagata [13], and for $R$ arbitrary by numerous persons. We list now a few of the results which we shall need to refer to in $\S 3$ and $\S 4$.

RESULT A. If $Q$ is a prime ideal of $R$, the set of all polynomials $f(x)$ in $R[X]$, all of whose coefficients belong to $Q$ is a prime ideal of $R[X]$, denoted by $Q[X]$. If $M$ is prime in $R[X]$ and if $P=R \cap M$, then $R[X] / M \cong \bar{R}[\tau]$ where $\bar{R}=R / P$ and $\tau$ is the $M$-residue of $X$. We have $P[X] \subseteq M$ and $M=P[X]$ if and only if $\tau$ is transcendental 
over $\bar{R}$. [20; Appendix I].

RESULT B. If $M_{1}, M_{2}, M_{3}$ are prime ideals of $R[X]$ with $M_{1} \subset M_{2} \subset M_{3}$, then $M_{1} \cap R \subset M_{3} \cap R$. Also, if $M_{1} \cap R=M_{2} \cap R$ then $M_{1}=\left[M_{1} \cap R\right][X]$. In particular if $M_{1} \neq(0)$ and $M_{1} \cap R=(0), M_{1}$ is a minimal prime of $R[X]$. [15; I; p. 505].

RESULT C. The element $f(X)=a_{0}+a_{1} X+\cdots+a_{n} X^{n}$ of $R[X]$ is a unit of $R[X]$ if and only if $a_{0}$ is a unit of $R$ and for $i>0, a_{i}$ is nilpotent in $R$. As a consequence, it follows that the Jacobson radical $R[X]$ is the set of nilpotent elements of $R[X]$. [16; p. 683].

3. The pseudo-radical of an integral domain. Suppose $R$ is a ring. If $R$ is not an integral domain then it is clear from the definition given in the introduction that the radical of $R$ and the pseudo-radical of $R$ coincide. Hence in considering the pseudo-radical in this section we restrict ourselves to the case when $R$ is an integral domain. We note that for a one-dimensional domain $D$ the pseudoradical of $D$ and the Jacobson radical of $D$ are equal. Theorem 3 shows how the property that the pseudo-radical of the domain $D$ is nonzero is reflected in the ideal theory of the polynomial domain $D[X]$. If $D$ is a domain with quotient field $K$ then by an overring of $D$ we mean any domain between $D$ and $K$.

Lemma 1. Suppose $D_{1}$ is a subring of the domain $D_{2}$ and that each nonzero ideal of $D_{2}$ intersects $D_{1}$ in a nonzero ideal of $D_{1}$. If the pseudo-radical $P_{1}^{*}$ of $D_{1}$ is nonzero, then the pseudo-radical $P_{2}^{*}$ of $D_{2}$ is also nonzero.

The hypothesis clearly implies that $P_{1}^{*} \subseteq P_{2}^{*} \cap D_{1}$, from which the conclusion follows.

Examples are easily constructed for which $P_{2}^{*}$ is nonzero but $P_{1}^{*}$ is zero - see, for instance, Example 1.

The hypothesis of Lemma 1 is satisfied if and only if each nonzero prime ideal of $D_{2}$ meets $D_{1}$ in a nonzero ideal. [12; 105]. In particular the hypothesis of Lemma 1 holds if $D_{2}$ is an overring of $D_{1}$ or if $D_{2}$ is integral over $D_{1}[19 ; 259]$. In the latter case we obtain a stronger conclusion than that of Lemma 1.

Lemma 2. If the domain $D_{2}$ is integral over its subring $D_{1}$, if $P_{i}^{*}$ is the pseudo-radical of $D_{i}$, and if $J_{i}$ is the Jacobson radical of $D_{i}$, then $J_{2} \cap D_{1}=J_{1}$ and $P_{2}^{*} \cap D_{1}=P_{1}^{*}$.

Both conclusions follow essentially from the "lying-over" theorem 
of Krull [9; 749] and its consequences. For example, to show $P_{1}^{*}=P_{2}^{*} \cap D_{1}$, we have $P_{1}^{*} \subseteq P_{2}^{*} \cap D_{1}$ by the proof of Lemma 1 . And if $t \in D_{1}-P_{1}^{*}, t \notin P$ for some nonzero prime $P$ of $D_{1}$. By the lying over theorem, there is a nonzero prime ideal $M$ of $D_{2}$ such that $P=M \cap D_{1}$. Hence $t \notin M$ and consequently $t \notin P_{2}^{*} \cap D_{1}$. Hence $P_{2}^{*} \cap D_{1} \subseteq P_{1}^{*}$ and equality holds.

Lemma 3. Let $D$ be a domain with quotient field $K$ and pseudoradical $P^{*}$. The following statements are equivalent.

(a) $P^{*} \neq(0)$.

(b) $K$ is a simple ring extension of $D$.

(c) $K$ is a finite ring extension of $D$.

(a) $\rightarrow(\mathrm{b})$ : If $t$ is a nonzero element of $P^{*}$, then $D[1 / t]=D_{\left\{t^{i}\right\}_{i=1}^{\infty}}$ is a quotient ring of $D$ such that each nonzero prime ideal of $D$ meets $\left\{t^{2}\right\}_{i=1}^{\infty}$. Therefore $(0)$ and $D[1 / t]$ are the only prime ideals of $D[1 / t] . \quad[19 ;$ p. 224]. Consequently $D[1 / t]$ is the field $K[19 ;$ p. 133] and (b) holds.

(b) $\rightarrow$ (c): trivial

(c) $\rightarrow$ (a): Let $K=D\left[t_{1}, \cdots, t_{n}\right]$ where $t_{i}=a_{i} / b_{i} ; a_{i}, b_{i} \in D$. Then clearly $K=D[1 / b]=D_{\left\{b^{i}\right\}_{i=1}^{\infty}}$ where $b=b_{1} b_{2} \cdots b_{n}$. If $M$ is a nonzero prime ideal of $D$ then $K=M K=M D_{\left\{b^{i}\right\}_{i=1}^{\infty}}$ so that $M$ meets $\left\{b^{i}\right\}_{1}^{\infty}$; hence $b \in M$ and $b \in P^{*}-\{0\}$, showing that (a) holds.

REMARK 1. If $D$ is a domain with pseudo-radical $P^{*} \neq(0)$ we let $d$ be a nonzero element of $P^{*}$. Then if $M$ is any nonzero proper prime ideal of $D, d \in M$ so that $M$ contains a minimal prime $P$ of $(d)$. [11; p. 12]. But by choice of $d, P$ is a minimal prime of $D$. Hence if the pseudo-radical of $D$ is nonzero, each nonzero proper prime of $D$ contains a minimal prime of $D$.

TheOREM 1. Suppose $D$ is a domain with pseudo-radical $P^{*}$. If $D$ is Noetherian, $P^{*}$ is nonzero if and only if $D$ is a onedimensional semi-local domain. If $D$ is a Krull domain, $P^{*}$ is nonzero if and only if $D$ is a semi-local principal ideal domain.

If $D$ is Noetherian, the conclusion that $D$ is one-dimensional and semi-local is the content of Theorom 4 of [2]. And the converse is obvious.

If $D$ is a Krull domain, let $\left\{v_{\alpha}\right\}_{\alpha \in_{A}}$ be a family of essential valuations for $D$ (see $\left[10 ; \mathrm{p}\right.$. 82] for terminology) with $v_{\alpha}$ associated with the minimal prime $P_{\alpha}$. If $t$ is a nonzero element of $P^{*}, v_{\alpha}(t)$ is nonzero for only finitely many of the $v_{\alpha}$ 's. But by choice of $t, v_{\alpha}(t)$ is positive for each $\alpha \in A$. Hence $A$ is a finite set and $D$ is an intersection of finitely many rank one discrete valuation rings. Hence $D$ is a semi- 
local principal ideal domain. ${ }^{1}$ Again it is clear that the pseudo-radical of such a $D$ is nonzero.

Theorem 1 shows that a Noetherian domain or a Krull domain having nonzero pseudo-radical is one-dimensional and has only finitely many maximal ideals. That one-dimensionality is not a necessary condition for the pseudo-radical to be zero may by seen by considering a valuation ring $D$ of $\operatorname{rank}>1$ such that $D$ has a minimal prime ideal. The following example shows that a domain with pseudo-radical nonzero may contain infinitely many maximal ideals.

Example 1. Let $A$ be the domain of all algebraic integers. The set $\left\{M_{\alpha}\right\}$ of maximal ideals of $A$ lying over a given maximal ideal $(p)$ of $Z$ is uncountable. [17; p. 31]. Moreover if $N=A-\left(\mathrm{U}_{\alpha} M_{\alpha}\right)$, $\left\{M_{\alpha} A_{N}\right\}$ is the set of maximal ideals of $A_{N}$. Because $A_{N}$ is one-dimensional, the pseudo-radical of $A_{N}$ is $\cap M_{\alpha} A_{N}=p A_{N} \neq(0)$.

Example 1 is more complicated than it need be to illustrate the fact that a domain with infinitely many maximal ideals may have nonzero pseudo-radical. But Example 1 illustrates some of the difficulties involved in characterizing Prüfer domains having nonzero pseudo-radical. And other than a restatement of Lemma 3 or Theorem 3 for the special case when $D$ is Prüfer we have, in fact, no such satisfactory characterization. The domain $A_{N}$ of Example 1 is onedimensionl and has the property that its finitely generated ideals are principal; hence $A_{N}$ is a domain with the $Q R$-property [5; p.99]. In particular $A_{N}$ is Prüfer. Theorem 2 represents our best result concerning arbitrary Prüfer domains having nonzero pseudo-radical.

Theorem 2. Suppose $D$ is a Prüfer domain with nonzero pseudoradical $P^{*}$. If $\left\{P_{\alpha}\right\}_{\alpha \in A}$ is the set of minimal prime ideals of $D$, the complete integral closure $D^{\prime}$ of $D$ is $\bigcap_{\alpha} D_{P_{\alpha}}$; therefore $D^{\prime}$ is completely integrally closed.

We shall use in the proof the following result which appears in an unpublished paper of the author, On the strong integral closure of an integral domain.

If $D_{2}$ is an overring of the domain $D_{1}$ and if the conductor of $D_{1}$ in $D_{2}$ is nonzero then $D_{1}$ and $D_{2}$ have the same complete integral closure.

Hence if we show the conductor $C$ of $D$ in $\cap D_{P_{\alpha}}=D^{*}$ is nonzero,

1) This result should be known though we are unable to give an explicit reference for the proof. Its validity follows from the following: By Theorem 11.11 of [14], $D$ is an almost Dedekind domain with only finitely many maximal ideals. By Theorom 3 of [3], $D$ is a semi-local principal ideal domain. 
then $D$ and $D^{*}$ have the same complete integral closure. But $D^{*}$ is an intersection of rank one valuation rings, each of which is completely integrally closed $[8 ; \mathrm{p} .170]$, so $D^{*}$ is completely integrally closed, and $D^{*}=D^{\prime}$ as asserted.

We show then that $P^{*} \subseteq C$. Thus let $p \in P^{*}$ and let $t \in D^{*}$. We show $p t \in D$.

If $\left\{M_{\beta}\right\}$ is the set of maximal ideals of $D$ it suffices to show $p t \in D_{M_{\beta}}$ for each $\beta$. [20; p. 94]. Let $P_{\beta}$ be the minimal prime of $D$ contained in $M_{\beta}$. Since $t \in D_{P_{\beta}}, t=a / b$ where $a \in D, b \in D-P_{\beta}$. If now $b \notin M_{\beta}, t \in D_{M_{\beta}}$ so that $p t \in D_{M_{\beta}}$. If $b \in M_{\beta}$ then we have

$$
p D_{M_{\beta}} \subseteq P_{\beta} D_{M_{\beta}} \subset b D_{M_{\beta}}
$$

it follows that $p / b \in D_{M_{\beta}}$ and $(p / b) a=p t \in D_{M_{\beta}}$. This completes our proof.

Finally, if $D$ is a domain having nonzero pseudo-radical $P^{*}$, we observe how this fact is reflected in the ideal theory of $D[X]$.

Theorem 3. Suppose $D$ is a domain with pseudo-radical $P^{*}$, $X$ is an indeterminate over $D$, and $K$ is the quotient field of $D$. The following statements are equivalent.

(a) $P^{*} \neq(0)$.

(b) there is a linear polynomial $f(X) \in D[X]$ such that $(f(X))$ is a maximal and minimal prime ideal in $D[X]$.

(c) there exists a prime ideal $M$ of $D[X]$ such that $M$ is both maximal and minimal in $D[X]$.

(d) there exists a maximal ideal of $D[X]$ lying over (0) in $D$.

(a) $\rightarrow(b)$ : By Lemma 3, (a) implies $K=D[1 / t]$ for some nonzero element $t$ of $D$. If then $f(X)=t X-1$, we have $D[X] /(t X-1) \cong$ $D[1 / t]=K$. Hence $P=(t X-1)$ is a maximal ideal of $D[X]$.

If $P_{1}$ is a prime ideal properly contained in $P, P_{1}=A P$ for some ideal $A$ of $D[X]$ since $P$ is principal. Since $P \supset P_{1}$ this implies $A \subseteq P_{1}=A P \subseteq A$. Thus $P_{1}=P_{1} P=P_{1} P^{2}=\cdots$ and $P_{1} \subseteq \cap_{n=1}^{\infty} P^{n}=$ $\cap{ }_{n=1}^{\infty}\left(f^{n}(X)\right)$, which is clearly zero. Hence $P_{1}=(0), P$ is minimal, and (b) holds.

$(b) \rightarrow(c)$ : trivial.

$(c) \rightarrow(d)$ : Suppose $M$ is both maximal and minimal in $D[X]$ and let $P=M \cap D . \quad P[X] \subseteq M$ and $P[X]$ is not maximal in $D[X]$ by Results $C$ and $A$. Hence $M \supset P[X] \supseteqq(0)$. By the minimality of $M$ it follows that $P[X]=(0)$ and hence that $P=(0)$ and $(d)$ holds.

$(d) \rightarrow$ (a): We let $M$ be a maximal ideal of $D[X]$ such that $M \cap D=(0)$. Then $D[X] / M \cong D / D \cap M[\tau] \cong D[\tau]$ is a field; $\tau$ the $M$-residue of $X$. This implies $\tau$ is algebraic over $D$ so that $d \tau$ is integral over $D$ for some nonzero $d \in D$. [18; 78]. Then $D[d \tau]$ has 
quotient field $D[\tau]=D[d \tau][\tau]$. By Lemma 3, the pseudo-radical of $D[d \tau]$ is nonzero. By Lemma $2, P^{*} \neq(0)$ also.

To prove $(d) \rightarrow$ (a) we could have appealed to a theorem of Nagata [13; I; p. 85], proved using different methods, which shows that (d) implies condition (b) of Lemma 3.

4. A generalization of a theorem of Goldman. Let $R$ be a ring and let $M$ be a prime ideal of the polynomial ring $R[X]$. If $P=M \cap R$ we seek a relation between $\left({ }^{*}\right)$ and $\left({ }^{* *}\right)$ :

$\left.{ }^{*}\right) \quad P$ is an intersection of maximal ideals of $R$.

(**) $\quad M$ is an intersection of maximal ideals of $R[X]$. Since $R[X] / M \cong \bar{R}[\tau]$ where $\bar{R}=R / P$ and $\tau$ is the $M$-residue of $X$, we see that $\left({ }^{* *}\right)$ holds if and only if the Jacobson radical of $\bar{R}[\tau]$ is zero. And $\left(^{*}\right)$ is equivalent to the condition that the Jacobson radical of $\bar{R}$ is zero. By Result $A, \tau$ is transcendental over $\bar{R}$ if and only if $M=P[X]$. And by Result $C, \tau$ transcendental over $\bar{R}$ implies the Jacobson radical of $\bar{R}[\tau]$ is zero. Hence $\left({ }^{* *}\right)$ does not imply $\left(^{*}\right)$ in general. In fact, Theorem 3 shows that if the pseudo-radical of the domain $D$ is nonzero, then there is a maximal ideal $M$ of $D[X]$ such that $\left(^{*}\right)$ is not valid for $M \cap D=(0)$.

We turn to the case when $\bar{R}[\tau]$ is algebraic over $\bar{R}$ - - that is, the case where $M \supset P[X]$. Here $d \tau$ is integral over $\bar{R}$ for some nonzero $d \in \bar{R}$. Further $\bar{R}$ has Jacobson radical zero if and only if $\bar{R}[d \tau]$ has Jacobson radical zero. Noting that $\tau$ is in the quotient field of $\bar{R}[d \tau]$ and that $\bar{R}[d \tau][\tau]=\bar{R}[\tau]$ it is then apparent that our earlier question concerning $(*)$ and $(* *)$ is equivalent to a consideration of this question: Let $D$ be a domain and $t$ be an element of the quotient field of $D$ such that $D$ is integrally closed in $D[t]$. What is the relation between these statements?

(\#) The Jacobson radical of $D$ is zero.

(\#\#) The Jacobson radical of $D[t]$ is zero. Our earlier observations concerning $(*)$ and $\left({ }^{*}\right)$ show that $(\# \#)$ does not imply $(\#)$ in general. We shall show, however, that (\#) implies (\#\#). From this it will follow that $\left({ }^{*}\right)$ implies $(* *)$; then Goldman's Theorem 3 of [6] follows as a corollary. We first prove one preliminary lemma.

LeMma $4 .^{2}$ Suppose $R$ is a commutative ring with Jacobson radical (0). If $A=\left\{a_{1}, a_{2}, \cdots, a_{n}\right\}$ is a finite set of regular elements of $R$, the intersection of all maximal ideals of $R$ not meeting $A$ contains no nonzero regular element. If "Jacobson radical" is replaced by "pseudo-radical" and "maximal" is replaced by "nonzero prime" the

2) For the proof of Lemma 4 as well as for several other helpful suggestions concerning the preparation of this paper, the author is grateful to W. J. Heinzer. 
statement obtained is also valid.

We prove the first statement of the lemma. Let $r$ be a nonzero regular element of $R$. By hypothesis $x=r a_{1} \cdots a_{n}$ is nonzero so there is a maximal ideal $M$ of $R$ such that $x \notin M$. Hence $r \notin M$ and no $a_{i}$ is in $M$; therefore $r$ does not belong to the intersection of the maximal ideals of $R$ not meeting $A$.

THeOREM 4. If $D$ is a domain with quotient field $K$ and if the Jacobson radical of $D$ is (0) then for $t \in K, D[t]$ has Jacobson radical zero.

We let $t=a / b ; a, b \in D$. If $D_{1}=D[t]$ and if $\left.D_{2}=D[1 / b]=D_{\{b i}\right\}_{i=1}^{\infty}$, then $D \subseteq D_{1} \subseteq D_{2}$. If $\left\{M_{\alpha}\right\}$ is the set of maximal ideals of $D$ not containing $b$, Lemma 4 shows $\cap M_{\alpha}=(0)$. For each $\alpha$ we have $M_{\alpha}=$ $M_{\alpha} D_{2} \cap D \supseteqq M_{\alpha} D_{1} \cap D \supseteqq M_{\alpha}$; hence $M_{\alpha}=M_{\alpha} D_{1} \cap D$ for each $\alpha$. Consequently $M_{\alpha} D_{1} \subset D_{1}$ so that $M_{\alpha} D_{1}$ is contained in a maximal ideal $P_{\alpha}$ of $D_{1}$. By the maximality of $M_{\alpha}$ in $D$, we must have $P_{\alpha} \cap D=M_{\alpha}$ for each $\alpha$. Thus $\left(\cap P_{\alpha}\right) \cap D=\cap\left(P_{\alpha} \cap D\right)=\cap M_{\alpha}=(0)$. Because $D_{1}$ is an overring of $D$, this implies $\cap P_{\alpha}=(0)$; in particular the intersection of all maximal ideals of $D_{1}$ — that is, the Jacobson radical of $D_{1}$ —is zero.

In view of our remarks preceding Lemma 4 , the next two corollaries follow immediately from Theorem 4.

CoROLlaRY 1. If $\tau$ is algebraic over the domain $D$, if $D[\tau]$ is an integral domain, and if the Jacobson radical of $D$ is (0), then the Jacobson radical of $D[\tau]$ is $(0)$.

COROLlaRY 2. Let $M$ be a prime ideal of the polynomial domain $D[X]$ over the domain $D$, and let $M \cap D=P$. If $M=P[X], M$ is an interesection of maximal ideals of $D[X]$. If $M \supset P[X]$ and if $P$ is an intersection of maximal ideals of $D, M$ is an intersection of maximal ideals of $D[X]$.

COROLlaRY 3. (Goldman) Let $X$ be an indeterminate over the ring $R . R[X]$ is a Hilbert ring if and only if $R$ is a Hilbert ring.

From the definition of a Hilbert ring, homomorphic images of Hilbert rings are again Hilbert. Thus if $R[X]$ is a Hilbert ring, so is $R[X] /(X) \cong R$. The converse follows from Corollary 2 .

It follows from Corollary 1 and from Result $\mathrm{C}$ that if the domain 
$D$ has Jacobson radical zero then $D_{1}$ has Jacobson radical zero where $D_{1}$ is any domain which is a finite ring extension of $D$. The hypothesis that $D_{1}$ be a domain is necessary as may be seen by taking $D=Z$ and $D_{1}=Z[X] /\left(X^{2}\right)$. Also, it is clear that Corollary 3 , as well as most of our other results could be stated for polynomial rings in finitely many indeterminates.

In conclusion we consider what can be said about the ideal structure of a domain $D$ such that for some element $t$ of the quotient field of $D, D[t]$ has Jacobson radical (0). Up to this point what we have observed is that we cannot conclude in general that $D$ has Jacobson radical zero. We now note that by Lemma 1 it is clear that if $D[t]$ is not the quotient field of $D$ then $D$ has pseudo-radical zero. If $D$ is finite-dimensional we nave a partial converse of this result.

Theorem 5. If the finite-dimenisonal domain $D$ has Jacobson radical nonzero and pseudo-radical zero, there exists an overing $D[t]$ of $D$ such that $D[t]$ has Jacobson radical zero.

We first observe that if $d$ is a nonzero element of $D$, the intersection of all nonzero primes of $D$ not containing $d$ is (0). This follows from Lemma 4. Hence if $z_{1}$ is a nonzero element of the Jacobson radical of $D$, what we have just noted implies $D_{1}=D\left[1 / z_{1}\right]$ is a domain with pseudo-radical (0). Also $D_{1}$ has dimension less than that of $D$ since $z_{1}$ belongs to each maximal ideal of $D$. If the Jacobson radical $J_{1}$ of $D_{1}$ is zero we have proved the theorem. If $J_{1} \neq(0)$ then $J_{1} \cap D \neq(0)$ also since $D_{1}$ is an overring of $D$. Hence if $z_{2}$ is a nonzero element of $J_{1} \cap D$ and if $D_{2}=D_{1}\left[1 / z_{2}\right], D_{2}$ has pseudo-radical zero, the dimension of $D_{2}$ is less than that of $D_{1}$, and $D_{2}=D\left[1 / z_{1} z_{2}\right]$ is a simple ring extension of $D$. If the Jacobson radical $J_{2}$ of $D_{2}$ is zero our proof is complete; if not, we continue the process. The process stops after finitely many steps since $D$ is finite-demensional and since for a onedimensional domain the Jacobson radical and the pseudo-radical coincide.

Corollary 4. Suppose $D$ is a finite-dimensional domain with nonzero Jacobson radical. In order that each simple overring $D[t]$ of $D$ which is not a field have nonzero Jacobson radical it is necessary and sufficient that the pseudo-radical of $D$ be nonzero.

Corollary 4 follows at once from Theorem 5 and the remarks preceding Theorem 5. The hypothesis of finite-dimensionality for $D$ cannot be dropped; if $D$ is a valuation ring having no minimal prime ideal, then each simple overing $D[t]$ of $D$ has nonzero Jacobson radical, but the pseudo-radical of $D$ is $(0)$. 


\section{REFERENCES}

1. I. S. Cohen and A. Seidenberg, Prime ideals and integral dependence, Bull. Amer. Math. Soc. 52 (1946), 252-261.

2. Robert W. Gilmer, Jr., Commutative rings containing at most two prime ideals, Michigan Math. J. 10 (1963), 263-268.

3. - Integral domains which are almost Dedekind, Proc. Amer. Math. Soc. 15 (1964), 813-818.

4. - On the strong integral closure of an integral domain, (to appear in Aust. J. Math.).

5. Rebert W. Gilmer, Jr. and Jack E. Ohm, Integral domains with quotient overrings, Math. Ann. 153 (1964), 97-103.

6. Oscar Goldman, Hilbert Rings and the Hilbert Nullstellensatz, Math. Zeit. 54 (1951), 136-140.

7. Chr, U. Jensen, On characterization of Prüfer rings, Math. Scand. 13 (1963), 90-98.

8. W. Krull, Allgemeine Bewertungstheorie, J. reine angew. Math. 167 (1931), 160196.

9. —, Beiträge zur Arithmetik kommutativer Integritatsbereiche. III, Math. Zeit. 42 (1937), 745-766.

10. - Jacobsonsche Ringe, Hilbertscher Nullstellensatz Dimensionentheorie, Math. Zeit. 54 (1951), 354-387.

11. —, Über einen Hauptsatz der allgemeinen Idealtheorie, S. B. Heidelberg. Akad. Wiss. 2 (1929), 11-16.

12. N. H. McCoy, Rings and Ideals, Menasha, Wisconsin, 1948.

13. Masayoshi Nagata, A general theory of algebraic geometry over Dedekind domains I, Amer. J. Math. 78 (1956), 78-116; II, Amer. J. Math. 80 (1958), 382-420; III, Amer. J. Math. 81 (1959), 401-435.

14. - Local Rings, Interscience, New York, 1962.

15. A. Seidenberg, A note on dimension theory of rings $I$, Pacific J. Math. 3 (1953), 505-512; II, Pacific J. Math. 4 (1954), 603-616.

16. Ernst Snapper, Completely primary rings I, Ann. of Math. 52 (1950), 666-693.

17. E. Stiemke, Uber unendliche algebraische Zahlkörper, Math. Zeit 25 (1926), 9-39.

18. B. L. Van der. Waerden, Modern Algebra, V. II, Ungar, New York, 1950.

19. O. Zariski and P. Samuel, Commutative Algebra I, Van Nostrand, Princeton, 1958.

20. - Commutative Algebra II, Van Nostrand, Princeton, 1960.

Received August 23, 1965, This research was supported by National Science Foundation Grant GP-4127.

FLORIDA State University 


\title{
PACIFIC JOURNAL OF MATHEMATICS
}

\author{
EDITORS
}

\section{H. SAMELSON}

Stanford University

Stanford, California

J. P. JANS

University of Washington

Seattle, Washington 98105
J. DugundJI

University of Southern California

Los Angeles, California 90007

RICHARD ARENS

University of California

Los Angeles, California 90024

\section{ASSOCIATE EDITORS}

\section{E. F. BECKENBACH}

B. H. NEUMANN

F. WOLF

K. YosidA

\section{SUPPORTING INSTITUTIONS}

\author{
UNIVERSITY OF BRITISH COLUMBIA \\ CALIFORNIA INSTITUTE OF TECHNOLOGY \\ UNIVERSITY OF CALIFORNIA \\ MONTANA STATE UNIVERSITY \\ UNIVERSITY OF NEVADA \\ NEW MEXICO STATE UNIVERSITY \\ OREGON STATE UNIVERSITY \\ UNIVERSITY OF OREGON \\ OSAKA UNIVERSITY \\ UNIVERSITY OF SOUTHERN CALIFORNIA
}

\author{
STANFORD UNIVERSITY \\ UNIVERSITY OF TOKYO \\ UNIVERSITY OF UTAH \\ WASHINGTON STATE UNIVERSITY \\ UNIVERSITY OF WASHINGTON \\ AMERICAN MATHEMATICAL SOCIETY \\ CHEVRON RESEARCH CORPORATION \\ TRW SYSTEMS \\ NAVAL ORDNANCE TEST STATION
}

Mathematical papers intended for publication in the Pacific Journal of Mathematics should be typewritten (double spaced). The first paragraph or two must be capable of being used separately as a synopsis of the entire paper. It should not contain references to the bibliography. Manuscripts may be sent to any one of the four editors. All other communications to the editors should be addressed to the managing editor, Richard Arens at the University of California, Los Angeles, California 90024.

50 reprints per author of each article are furnished free of charge; additional copies may be obtained at cost in multiples of 50 .

The Pacific Journal of Mathematics is published monthly. Effective with Volume 16 the price per volume (3 numbers) is $\$ 8.00$; single issues, $\$ 3.00$. Special price for current issues to individual faculty members of supporting institutions and to individual members of the American Mathematical Society: $\$ 4.00$ per volume; single issues $\$ 1.50$. Back numbers are available.

Subscriptions, orders for back numbers, and changes of address should be sent to Pacific Journal of Mathematics, 103 Highland Boulevard, Berkeley 8, California.

Printed at Kokusai Bunken Insatsusha (International Academic Printing Co., Ltd.), No. 6, 2-chome, Fujimi-cho, Chiyoda-ku, Tokyo, Japan.

PUBLISHED BY PACIFIC JOURNAL OF MATHEMATICS, A NON-PROFIT CORPORATION

The Supporting Institutions listed above contribute to the cost of publication of this Journal, but they are not owners or publishers and have no responsibility for its content or policies. 


\section{Pacific Journal of Mathematics \\ Vol. 19, No. $2 \quad$ June, 1966}

Leonard Daniel Baumert, Extreme copositive quadratic forms . ......... 197

Fred James Bellar, Jr., Pointwise bounds for the second initial-boundary value problem of parabolic type ........................ 205

L. Carlitz and David Paul Roselle, Restricted bipartite partitions ........ 221

Robin Ward Chaney, On the transformation of integrals in measure space ........................................... 229

Colin W. Clark, An embedding theorem for function spaces ........... 243

Edwin Duda, A theorem on one-to-one mappings................ 253

Ben Fitzpatrick, Jr. and Donald Reginald Traylor, Two theorems on metrizability of Moore spaces.......................... 259

Allen Roy Freedman, An inequality for the density of the sum of sets of vectors in $n$-dimensional space ....................... 265

Michael Friedberg, On representations of certain semigroups .......... 269

Robert William Gilmer, Jr., The pseudo-radical of a commutative ring . . . . 275

Hikosaburo Komatsu, Fractional powers of operators ............... 285

Daniel Rider, Transformations of Fourier coefficients ................. 347

David Alan Sánchez, Some existence theorems in the calculus of variations ...................................... 357

Howard Joseph Wilcox, Pseudocompact groups............... 365

William P. Ziemer, Some lower bounds for Lebesgue area ............. 381 